\title{
Belphégor
}

\section{Fabio Gadducci, Leonardo Gori, Sergio Lama, Eccetto Topolino: Lo scontro culturale tra Fascismo e Fumetti}

\author{
Isabelle Antonutti
}

\section{(2) OpenEdition}

1 Journals

\section{Édition électronique}

URL : http://journals.openedition.org/belphegor/488

DOI : 10.4000/belphegor.488

ISSN : 1499-7185

Éditeur

LPCM

\section{Référence électronique}

Isabelle Antonutti, «Fabio Gadducci, Leonardo Gori, Sergio Lama, Eccetto Topolino : Lo scontro culturale tra Fascismo e Fumetti », Belphégor [En ligne], 12-1 | 2014, mis en ligne le 12 juin 2014, consulté le 22 septembre 2020. URL : http://journals.openedition.org/belphegor/488 ; DOI : https://doi.org/10.4000/ belphegor.488

Ce document a été généré automatiquement le 22 septembre 2020.

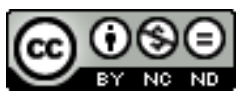

Belphégor est mis à disposition selon les termes de la Licence Creative Commons Attribution - Pas d'Utilisation Commerciale - Pas de Modification 4.0 International. 
Fabio Gadducci, Leonardo Gori, Sergio Lama, Eccetto Topolino : Lo scontro culturale tra Fascismo e Fumetti

Isabelle Antonutti

\section{RÉFÉRENCE}

Eccetto Topolino : Lo scontro culturale tra Fascismo e Fumetti, Fabio Gadducci, Leonardo Gori, Sergio Lama, Roma : Nicola Pesce Editore (NPE), 2011431 pp. ISBN 978-8-89714-104-4 
1 Cet ouvrage restitue l'histoire éditoriale de la bande dessinée italienne dans les années trente. L'intérêt précieux de cette recherche est d'explorer la relation entre le monde éditorial et la société politique. L'histoire de la bande dessinée a largement été relatée, les auteurs, les séries, les publications ont déjà été disséqués. Eccetto Topolino s'intéresse aux coulisses économiques, politiques et sociales du métier d'éditeur. Il restitue les rapports, le choc culturel, comme l'indique le sous-titre, entre cette industrie culturelle naissante et le régime fasciste. Les auteurs explorent des sources inédites issues de la Fondation Franco Fossati comme par exemple les archives de l'agent italien de la KFS, Guglielmo

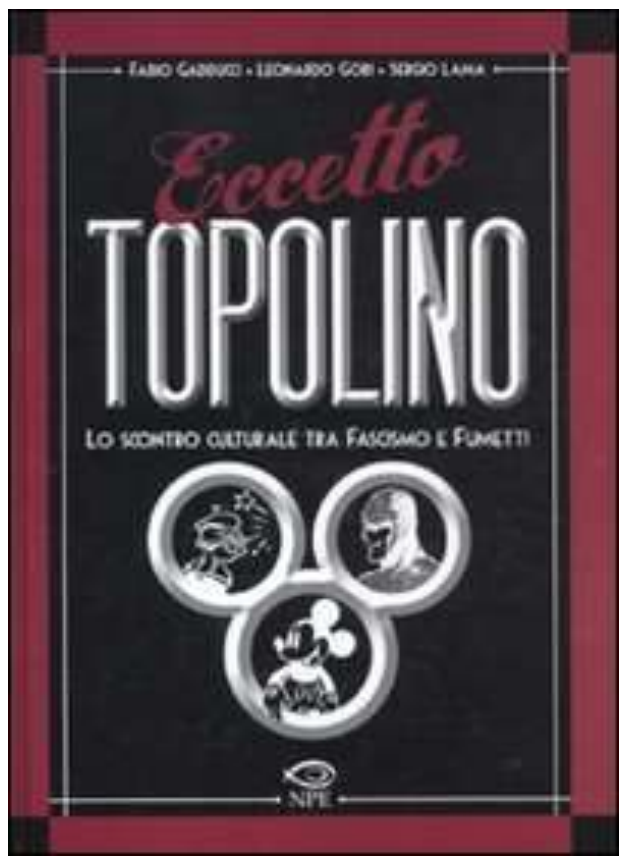
Emanuel. Ce journaliste, qui fut après guerre directeur du quotidien Il Corriere della sera, est au cœur des échanges entre les éditeurs italiens, les revendeurs américains et les représentants politiques. Les compétences professionnelles de Guglielmo Emanuel lui permettent de saisir les enjeux économiques et culturels de cette production graphique pour la jeunesse. Les lettres, les notes éclairent les relations croisées entre Benito Mussolini, Walt Disney, William R. Hearst, Arnoldo Mondadori et Mario Nerbini. Les auteurs utilisent aussi les archives privées de Federico Pedrocchi, un des pères du fumetto. Ces nouvelles sources sont croisées avec les Archives centrales de l'État et celles de la Fondation Mondadori.

2 Trois éditeurs, Lotario Vecchi, Giuseppe Nerbini et Arnoldo Mondadori sont particulièrement étudiés. Ils ont importé les comics puis créé le fumetto. L'ouvrage débute en 1932 avec l'illustré pour enfants Jumbo, publié par l'éditeur Vecchi et se termine à la fin de la seconde guerre mondiale. Pendant ces 13 ans, face au succès rencontré auprès des jeunes, de nombreux éditeurs commencent à publier des illustrés hebdomadaires comme L'Avventuroso ou Audace. Ces magazines sont conçus à partir de bandes dessinées importées d'Angleterre et surtout des États-Unis. Dans ce pays, la vente des productions graphiques est gérée par des syndicates. En 1912, William R. Hearst avait créé le King Features Syndicate (KFS) qui devient le principal diffuseur vers l'Europe. Il exporte des séries de personnages, désormais mondialement connus, comme Tarzan, Flash Gordon ou Mandrake.

3 L'ouvrage relate les conditions d'exploitation de la bande dessinée d'importation américaine, les comics, et de son adaptation en Italie. Mais surtout, il insiste sur les relations tumultueuses de ce secteur éditorial avec le régime fasciste. La publication des comics était sérieusement surveillée, particulièrement après la création du ministère de la Culture populaire en 1938. Certaines reproductions comparent les dessins américains originaux et les planches retouchées en Italie, les femmes sont rhabillées, les armes sont cachées. Les éditeurs s'adaptent aux interdictions et s'autocensurent. Toutefois, le régime fasciste est aussi équivoque car les importations la firme Walt Disney ont échappé à la censure gouvernementale. Le titre de l'ouvrage 
Eccetto Topolino (excepté Mickey) y fait référence. Les auteurs reviennent longuement sur cette exception et ses conséquences. Pourquoi donc cette permission accordée à Mickey et consorts? La légende dit que ces histoires plaisaient à la famille Mussolini mais la réalité est plus complexe. Cette dérogation résulte aussi de l'influence d'Arnoldo Mondadori qui avait la confiance du régime. Par ailleurs, KFS savait aussi entretenir des relations utiles auprès des notables fascistes. Cette singularité a donné naissance à une relation particulière avec l'œuvre de Disney : l'Italie est le seul pays à avoir transformé les noms des héros, Topolino pour Mickey, par exemple, et à avoir eu l'autorisation de créer des histoires originales.

4 L'ouvrage, très soigné, se divise en neuf chapitres qui suivent une progression chronologique des années trente à la fin de la seconde guerre mondiale. Ce beau volume cartonné est agréablement mis en page avec de nombreuses illustrations, dont beaucoup de copies de courrier et documents administratifs. Une section d'interviews, une bibliographie très pertinente et la liste des illustrés autorisés complètent l'étude centrale. Au-delà de l'intérêt spécifique sur l'histoire de l'édition italienne, cette recherche apporte une contribution fort intéressante sur les dimensions politiques et économiques des industries culturelles.

$5 \quad$ Les trois auteurs sont des passionnés de bandes dessinés et ils sont issus d'univers différents. Fabio Gaducci est professeur d'informatique auprès de l'université de Pise. Leonardo Gori est romancier et il anime un excellent blog sur l'histoire de la bande dessinée Fumetti classici' ${ }^{1}$ Sergio lama est bibliographe et collectionneur. Ils allient leurs qualités respectives, l'ouvrage est d'une minutieuse rigueur tout en gardant rythme et suspense.

\section{NOTES}

1. http://annitrenta.blogspot.fr/ 